\title{
Detection of the initial region of the current transformer core saturation
}

\author{
I. Odinaev ${ }^{1}$, Andrew V. Pazderin ${ }^{1}$, Pavel V. Murzin ${ }^{1}$, Valeriy A. Tashchilin ${ }^{1}$, Vladislav O. \\ Samoylenko ${ }^{1}$ and B. Ghoziev ${ }^{1}$ \\ ${ }^{1}$ Department of Automated Electrical Systems \\ Ural Federal University \\ Mira str., 19, 620002 Yekaterinburg (Russia) \\ Phone/Fax number: +007 343 359-16-15, e-mail: ismoil.odinaev@urfu.ru, a.v.pazderin@urfu.ru, \\ p.v.murzin@urfu.ru, v.a.tashchilin@urfu.ru, vvsamoylenko@yandex.ru, b.n.goziev@urfu.ru.
}

\begin{abstract}
In the first part of the paper the detailed analysis of the existing current transformer (CT) saturation detection methods with their classification is presented. A new saturation detection method has been proposed in second part of the paper. Mathematical description of the method is given. In the next section of the paper a CT model with test scheme is presented. To identify the reliability of proposed method against nose and remanent flux density experiments have been produced in the fourth section. Comparative analysis between proposed and existing methods is also given. Finally, in conclusion a detailed description of the method is given regarding its behavior with respect to remanent flux and noise.
\end{abstract}

Key words. Current transformer (CT), saturation detection, flux density, unsaturated section.

\section{Introduction}

One of the main current measurement sources in electrical power systems are electromagnetic CTs. Information obtained from these CTs is used for both protection and metering systems. However, as shown in [1], during faults the core of such CTs can saturate and as a result a maloperation of the protection system (PS) may take place. According to [2] CT saturation affects sensitivity and time operation of PS as well as on the accuracy of the fault localization method.

In order to avoid the negative impact of CT saturation on the PS operation, it is proposed to use mathematical methods to detect CT saturation [3] - [16] with subsequent correction of the measured current [17] - [20]. From the point of digital signal processing view, the CT error compensation problem in saturation mode can be divided into three sub-problems - segmentation, selection, and filtering. Segmentation is an operation of dividing measured current into normal and transient sections (modes), selection is a choosing measured current samples connected to CT unsaturated section (US) and filtering is a correction of measured current samples connected to CT saturated section.

The most difficult of the listed ones are the selection and filtering sun-problems. Typically, the selection problem is solved using detection algorithms based on the methods of mathematical analysis (difference quotient) [3] - [11] and mathematical statistics [12]-[16].

The saturation detection method proposed in [3]-[10] operates based on difference function [18]. During testing of [18] it was identified that the method has a high sensitivity to white noise which influences on the method results. In order to avoid this effect, the authors of [9] and [10] using Savitzky-Golay filter [21] performed preliminary smoothing of measured current. However, as shown by the test results, the method is unable to provide detection during severe saturation. Moreover, if during saturation occurrence measured current has no sharp decline, the method failing to provide detection (Fig. 1,b).

In [11] for detecting CT saturation the difference function proposed in [22] is used. Difference between [18] and [22] functions is that the first one applies current derivative, which in saturation cases has a high splash, the last one based on US section predicts measured current samples in saturated section that allow to determine the difference between predicted and saturated samples. Based on the obtained information the saturation detection problem is solved. During testing the method in [22] it was discovered that the measured current with noise significantly effects on the method efficiency and as result it leads to the method efficiency losses.

In [12] based on two adjacent extreme points of the first derivative of the measured current, obtained last (last and penultimate), the following two criteria are formulated:

1. The length of timeline between these extreme points must be less than $10 \mathrm{~ms}$ i.e. $\left(\left|t_{\text {last }}-t_{\text {penultimate }}\right|<10 \mathrm{~ms}\right)$.

2. The ratio of these extreme points is greater than 1 i.e. $\left(\left|\frac{i_{\text {last }}}{i_{\text {penultimate }}}\right|>1\right)$. 
When criteria 1 and 2 are simultaneously true, the CT saturation is fixed. The justification for the method is that differentiation of the measured current reduces its DC component by a factor less than 1 in the power of the derivative order. Thus, due to multiple differentiation of the measured current, its constant component is reduced to an insignificant level. By eliminating the filtering time, the method can detect CT saturation with a time offset of $t$ $=1.2 \mathrm{~ms}$, that results to an additional delay in the PS operation. However, the method provides only saturation existence in the CT core but not the end determination of the US section. This complicates the selection of well measured samples of secondary current.

To detect CT saturation in the two-winding power transformer differential protection scheme the detection method is proposed in [13] and [14]. Based on the Pearson correlation coefficient the method establishes connection between currents on high and low sides of the power transformer. It is important to note that usage of the method in the busbar differential protection scheme requires processing of large amount of data that leads to an increase of computational efforts. Moreover, solution of the saturation detection problem by the method will be complicated in the case of a single CT.

Authors in [15] propose a method that is based on current behavior in the differential protection scheme. During external faults resulting to CT saturation the shape of the differential current has two specific sections. The first of them corresponds to US section and the second to saturated one. It is worth noting that the method allows to detect saturation of those CTs that included in the differential protection. Furthermore, the method detects CT saturation in the protection scheme but not the definite saturated CT. In this case the filtering problem of the measured current with distorted sections will be complicated.

The method proposed in [16] is based on the analysis of the location density of the measured current samples. In severe CT saturation the measured current samples are located close to time axis and in CT normal conditions these samples near the mentioned axis have a wide scatter. Such scatter is due to the fact that measured current samples are located on the linear part of sine current in normal conditions. Fig. 1 shows the measured current samples (Meas.) with their distribution density and reference current (Ref.). It is worth noting that the method can detect a severe CT saturation or a saturation that severely limits the measured current samples and as a result these samples are located close to time axis, Fig. 1, a. During CT saturation that leads to slow offsetting of the measured current from reference, it is impossible to detect saturation by the method, Fig. $1, b$.

In this study on the basis of monitoring the flux density (MFD) in the CT core a solution of the saturation detection problem was proposed. More detailed description of the detection pressure is given in the next section.

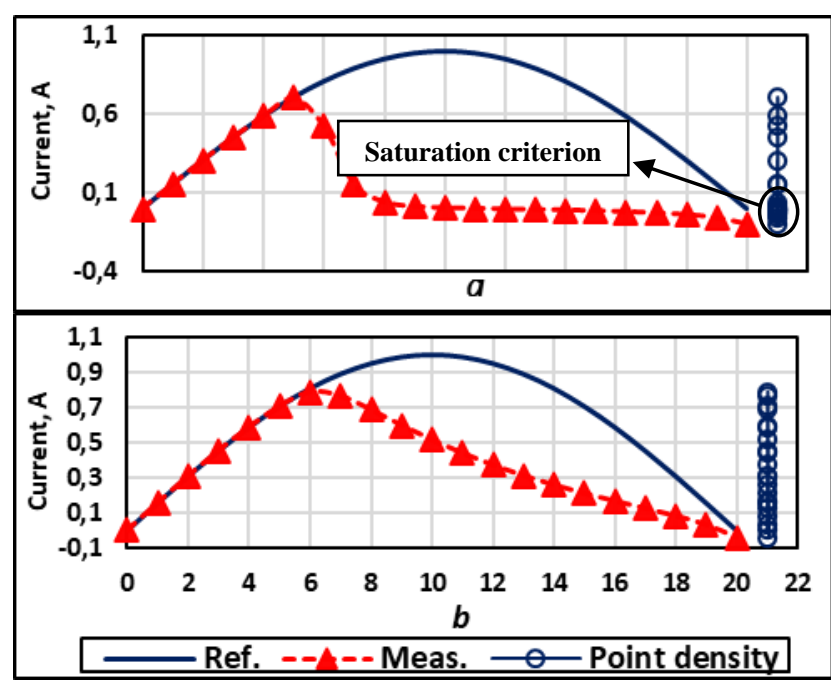

Fig. 1. Graphic interpretation of the saturation detection method [16]

\section{CT saturation detection based on MFD}

Within this section the detailed description of the MFD method is proposed. To implement the method, the following CT parameters are required to be known:

- magnetizing curve $H=f(\lambda)$;

- secondary circuit resistance $R_{2}, \Omega$;

- secondary circuit resistance $L_{2}, \Omega \cdot s / \mathrm{rad}$;

- path length $l, m$;

- cross section, $s, m^{2}$;

- turning number of the secondary winding, $w_{2}$.

The detection is based on the fact that when the CT core enters the saturation region its flux density $\lambda$ changes quickly and instead of a sine shape (i.e. unsaturated mode) it has stationary areas that resemble a trapezoid. In these areas, the value of the derivative and variance of the $\lambda$ sharply decreases. For presentation purposes, the upper plots of the Fig. 2 and Fig. 3 show reference (Ref.) and measured (Meas.) currents of the CT, the lower plots show the change in the reference flux density $\lambda$. As can be seen from the lower plots, in normal conditions the $\lambda$ has a sine shape and when the CT core saturation occurs its shape takes a form resembling a trapezoid. The second case means the measure of the flux density variance $\sigma$ in relation to its population mean $\bar{\lambda}_{e}$ in saturated areas is greatly reduced which can be seen on the lower plot on the Fig. 3.

To estimate the flux density variance $\sigma$ its calculated $\lambda_{c}$ must be known. According to [23] the $\lambda_{c}$ can be obtained using expression (1).

$$
\begin{aligned}
& B(n)=\frac{R_{2}}{w_{2} s}\left[i_{2}(n)+i_{2}(n-1)\right] \cdot \Delta t+ \\
& +\frac{L_{2}}{w_{2} s} \cdot\left[i_{2}(n)-i_{2}(n-1)\right]+B(n-1)
\end{aligned}
$$




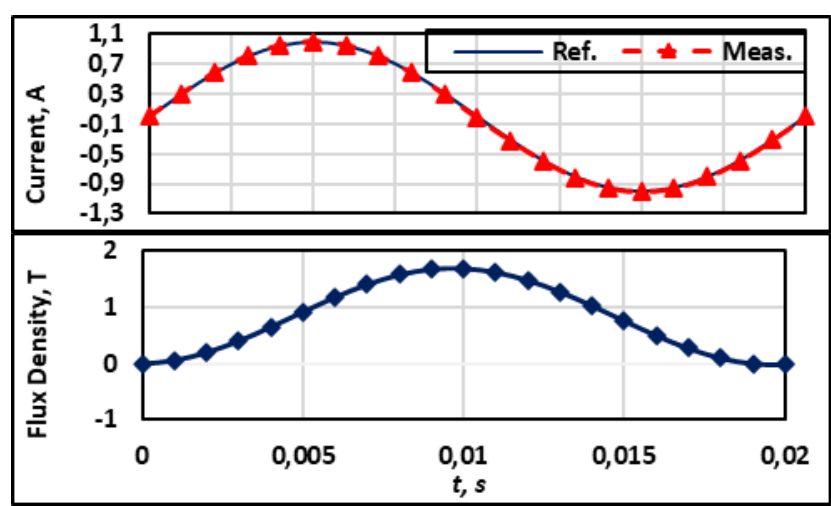

Fig. 2. Upper plot - reference and measured currents; lower plot - the flux density $\lambda$ in normal conditions

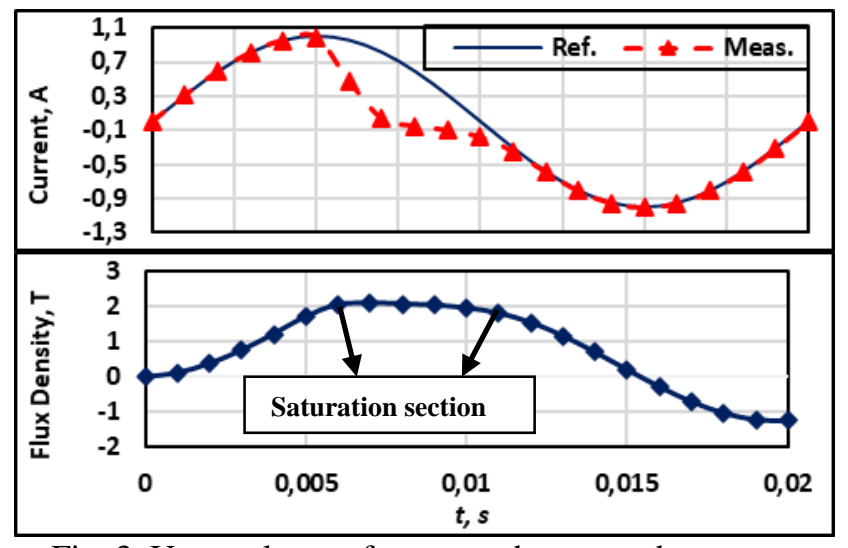

Fig. 3. Upper plot - reference and measured currents; lower plot - the flux density $\lambda$ in CT saturation

In (1), due to unknown initial flux density which is represented as $\lambda(n-1)$, there will be a shift between the reference $\lambda$ and calculated $\lambda_{c}$. However, the shape of the calculated and reference flux density will match exactly which is an important fact for the MFD method. As the MFD method estimates the location of $\lambda_{c}$ samples relative to its population mean $\bar{\lambda}_{e}$, excluding the initial flux density $\lambda(n-1)$ in (1) will not impact on the efficiency of the method.

Thus, knowing the behavior of the flux density in the CT core, its deviation $\sigma$ from the population mean $\bar{\lambda}_{e}$ can be calculated using expression (2).

$\sigma(n-N)=\frac{1}{N-1} \sum_{m=1}^{N}\left|\lambda(n-m)-\bar{\lambda}_{e}\right|^{2}$,

where $n$ is the number of the current reading of the $\lambda_{c} ; m$ is an integer that serves as a window function; $N$ is the length of the window; $\bar{\lambda}_{e}$ is the population mean.

The $\bar{\lambda}_{e}$ is calculated by equation (3).

$\bar{\lambda}_{e}=\frac{1}{N} \sum_{m=1}^{N} \lambda(n-m)$.

It is worth noting that the length of the window $N$, including in deviation calculation, is a crucial factor of the
MFD method. Varying $N$ allows to change the method sensitivity against the various levels of CT core saturation.

Knowing the deviation of the calculated flux density $\sigma$ within the interval $N$, it is possible to determine the presence of CT core saturation. In order to do so, it is necessary to compare $\sigma$ with a threshold value $T h$, which corresponds to the slope of the magnetizing curve section in the range from $k$ to $m$. The point $k$, located at the beginning of the section $H=f(\lambda)$, corresponds to the first sample of the magnetization curve and the point $m$ corresponds to the maximum value of the $\lambda$ at the rated load of the CT in the case of peak load operation of a grid.

To calculate $T h$ the usage of the $\lambda(n)$ values, located on the magnetizing curve section in the $k$ and $m$ range, is required. For presentation purposes, the Fig. 4 shows the magnetizing curve on the basis of which $k$ and $m$ are determined.

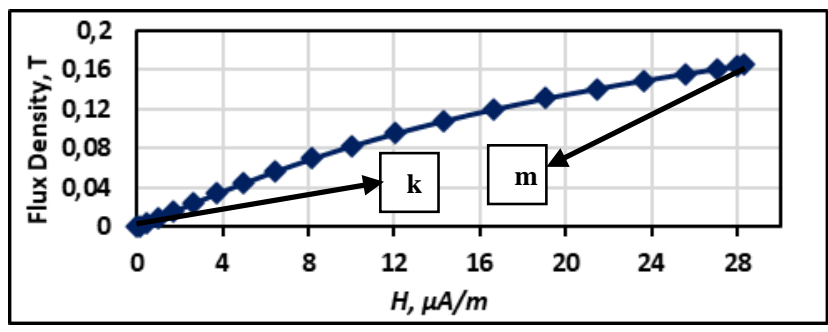

Fig. 4. CT magnetizing curve

The $T h$ is calculated according to equation (4).

$T h=\frac{\sum_{k=1}^{m}\left(\lambda_{k}-\bar{\lambda}_{e}\right)^{2}}{m}$.

The occurrence of saturation is determined in accordance with (5).

$\sigma(n)<T h$

The window length $N$ must be selected so that during faults not leading to $\mathrm{CT}$ core saturation the maloperation of the MFD is blocked, i.e. $T h$ would not exceed the $\sigma(n)$. To avoid maloperation of the method under normal and peak load conditions of a grid, the start of the method is triggered by the following expression:

$C>\sqrt{2} \cdot I_{\max }$,

where $I_{\max }$ is an RMS value of current according to peak load conditions of a grid; $C$ is the predicted amplitude of the measured current.

The $C$ prediction can be explained as follows: let there be some sine signal with its set of the instantaneous values and analytical expression (7).

$i(t)=C \cdot \sin \left(\omega t+\varphi_{0}\right)$,

where $\omega$ is an angular rate; $\varphi_{0}$ is an initial phase.

Make an arbitrary measurement and set its measurement time $t=0$, then expression (7) can be rewritten as follows: 
$i(0)=C \cdot \sin \left(\varphi_{0}\right)$

Derivative of expression (7) is:

$i^{\prime}(t)=\frac{d i}{d t}=\omega C \cos \left(\omega \cdot t+\varphi_{0}\right)$.

At $t=0$ expression (9) cab be rewritten as:

$i^{\prime}(0)=\omega C \cos \left(\varphi_{0}\right)$.

The value of $i(0)$ is known it is an instantaneous value for which $t=0$, further it will be denoted as $i_{0}$, the derivative $i^{\prime}(0)$ will be denoted as $i_{0}^{\prime}$, and so the tangent of the slope angle, drawn between two points that located from left $i_{-1}$ and right $i_{+1}$ of the $i_{0}$, can be determined as following:

$i_{0}^{\prime}=\frac{d i}{d t} \approx \frac{\Delta i}{\Delta t}=\frac{i_{+1}-i_{-1}}{2 \Delta t}$,

where $\Delta t$ is a sampling step.

On the basis of the expressions (8) and (10) following equations can be received:

$\sin ^{2}\left(\varphi_{0}\right)+\cos ^{2}\left(\varphi_{0}\right)=1$

$\left(\frac{i_{0}}{C}\right)^{2}+\left(\frac{i_{0}^{\prime}}{\omega C}\right)^{2}=1$

$\left(i_{0}\right)^{2}+\left(\frac{i_{0}^{\prime}}{\omega}\right)^{2}=C^{2}$

$C=\sqrt{\left(i_{0}\right)^{2}+\left(\frac{i_{0}^{\prime}}{\omega}\right)^{2}}$.

Thus, based on US section the predicted amplitude of the measured current can be obtained. To improve the predict accuracy it is proposed to calculate the overage derivative $i^{\prime}{ }_{0}$ using 3-4 measured current samples related to US section.

\section{Test Model description}

To perform experiments allowing to determine the MFD ability a grid was modeled in Matlab/Simulink environment, that includes load, system S, transmission line and two-winding power transformer with CTs on both high (CT1) and low (CT2) sides. The installed load value was set equal to $70 \%$ of power transformer nominal rate, $\cos \varphi=0.8$. The configuration elements of the grid and their main parameters are presented on Fig. 5.

Modeling of CT1 and CT2 was performed on the basis of the Jiles-Atherton theory that considers hysteresis loop and losses on the CT core excitation [24], [25]. The JilesAtherton theory includes next parameters: density flux $\lambda$; magnetizing field $H$; effective field $H_{e}$; magnetization $M$ with its reversible $M_{r e v}$ and irreversible $M_{i r r}$ components as well as anhysteretic magnetization $M_{a n}$.

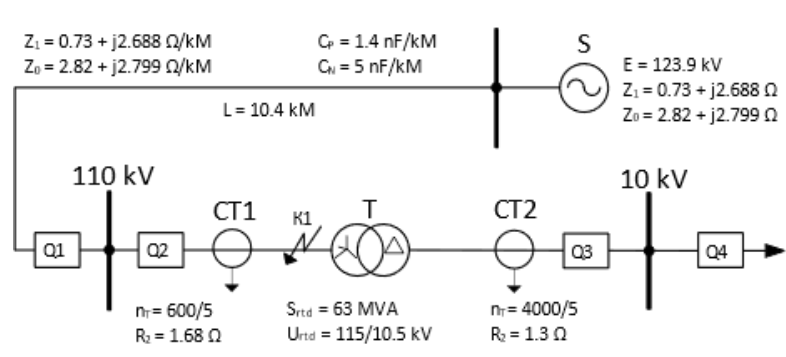

Fig. 5. Test model

\section{Testing of the MFD method}

In this section, the testing of the MFD method was performed. To determine the method sensitivity with respect to nose $(\delta)$ and level of the initial flux density $\lambda_{0}$ in the CT core, a $\delta$ and $\lambda_{0}$ were included in the measured current and in the CT core respectively. Including noise was performed by equation (16).

$i_{\text {meas }}(t)=i_{2}(t) \cdot\left(1+\delta_{1} \cdot \vartheta\right)+\max \left(i_{2}(t)\right) \cdot\left(\delta_{2} \cdot \vartheta\right)$,

where $i_{2}(t)$ is a measured current; $\delta_{1}$ and $\delta_{2}$ are priori specified values of the noise level; $\vartheta$ is a random number in the range of -1 to $1 . \vartheta$ has a uniform distribution.

\section{Checking the MFD sensitivity to a noise}

Within this experiment, based on the CT1 of the test model, checking the method sensitivity to a noise was provided. To do so a noi se with a level of $\delta_{1}=3 \%$ and $\delta_{2}$ $=1 \%$ was added to the measured current. Initial phase of the modeled fault $\varphi_{A O}$ was included for phase $A$ and changed in the range of $0^{\circ}$ to $90^{\circ}$. The initial phase range $\left(0^{\circ}-90^{\circ}\right)$ was chosen based on dividing the entire cycle of the sine current into 4 parts, i.e. $0^{\circ}-90^{\circ}, 90^{\circ}-180^{\circ}, 180^{\circ}$ $-270^{\circ}$ and $270^{\circ}-360^{\circ}$. The simulation of the other three parts was not performed under the assumption that their influence on the fault transient is similar or reflection of the chosen part. The CT core initial flux density $\lambda_{0}$ for all $\mathrm{CT}$ was excluded and specified $\lambda_{0}=0 T$ Fig. 6 shows the results of experiment, where reference and measured currents and the result of the MFD method for phases $A$ and $B$ during a fault between them are presented. As in this case of fault the CT of the third phase operates with normal conditions, its currents and saturation detection were not performed.

It is clear seen from Fig. 6 that a noise in the measured current has no negative impact on the result of MFD operation. It is worth to note that the variation of initial phase $\varphi_{A 0}$ has no impact on the MFD as well. The last four plots of the Fig. 6 show that during CTs cores saturation the shape of the measured current in the phase $B$ is significantly different from that in the phase $A$. Nevertheless, the method allows to detect the start of the CT saturation in both cases. 


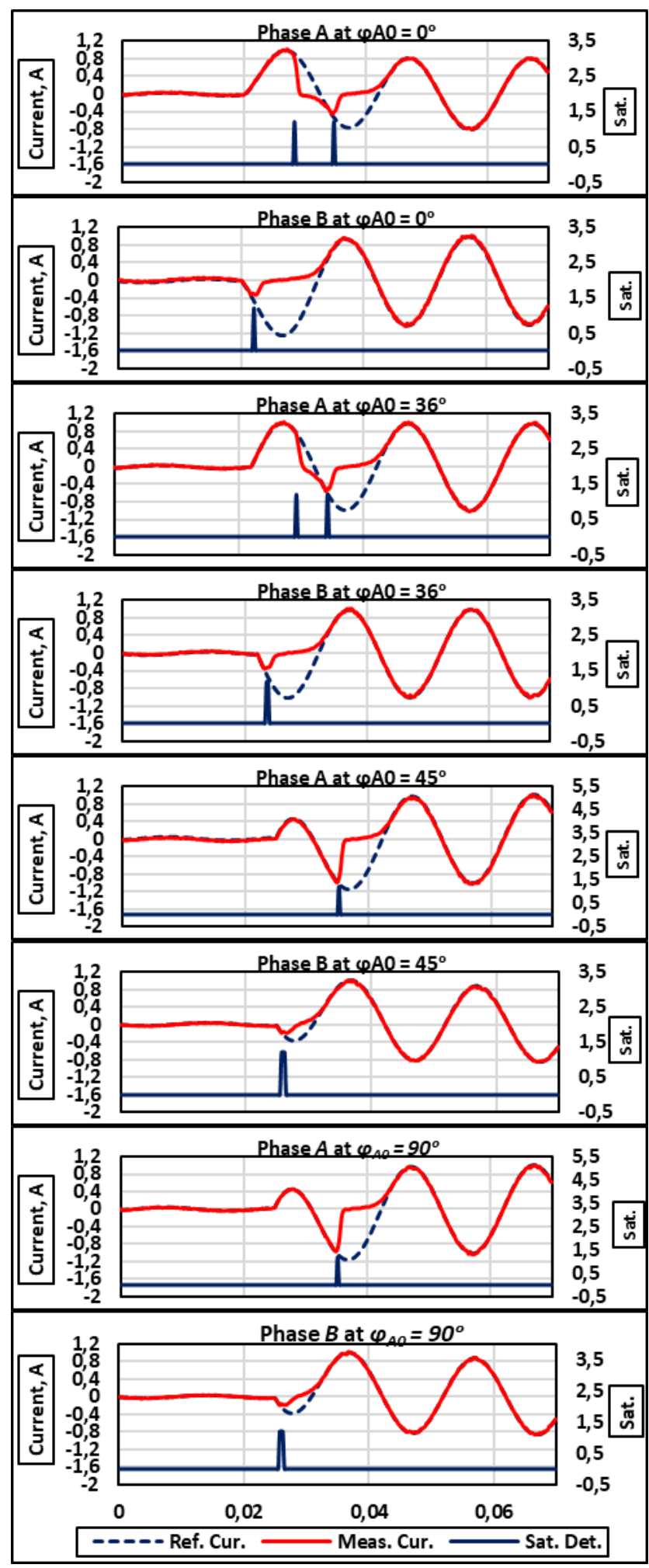

Fig. 6. CT currents and its saturation detection with variation of $\varphi_{A O}$

\section{Checking the MFD sensitivity to an initial flux density}

In this experiment it was intended to check the MFD method sensitivity to the CT core initial flux density $\lambda_{0}$ with various levels. Variation of the $\lambda_{0}$ was performed in the range of -0.5 to $1.8 T$ only in the CT core established on the phase $A$. An initial phase was a constant and specified as $\varphi_{A O}=0^{\circ}$. Fig. 7 shows the reference and measured currents as well as the result of the MFD method for phase $A$ during fault between phases $A$ and $B$.

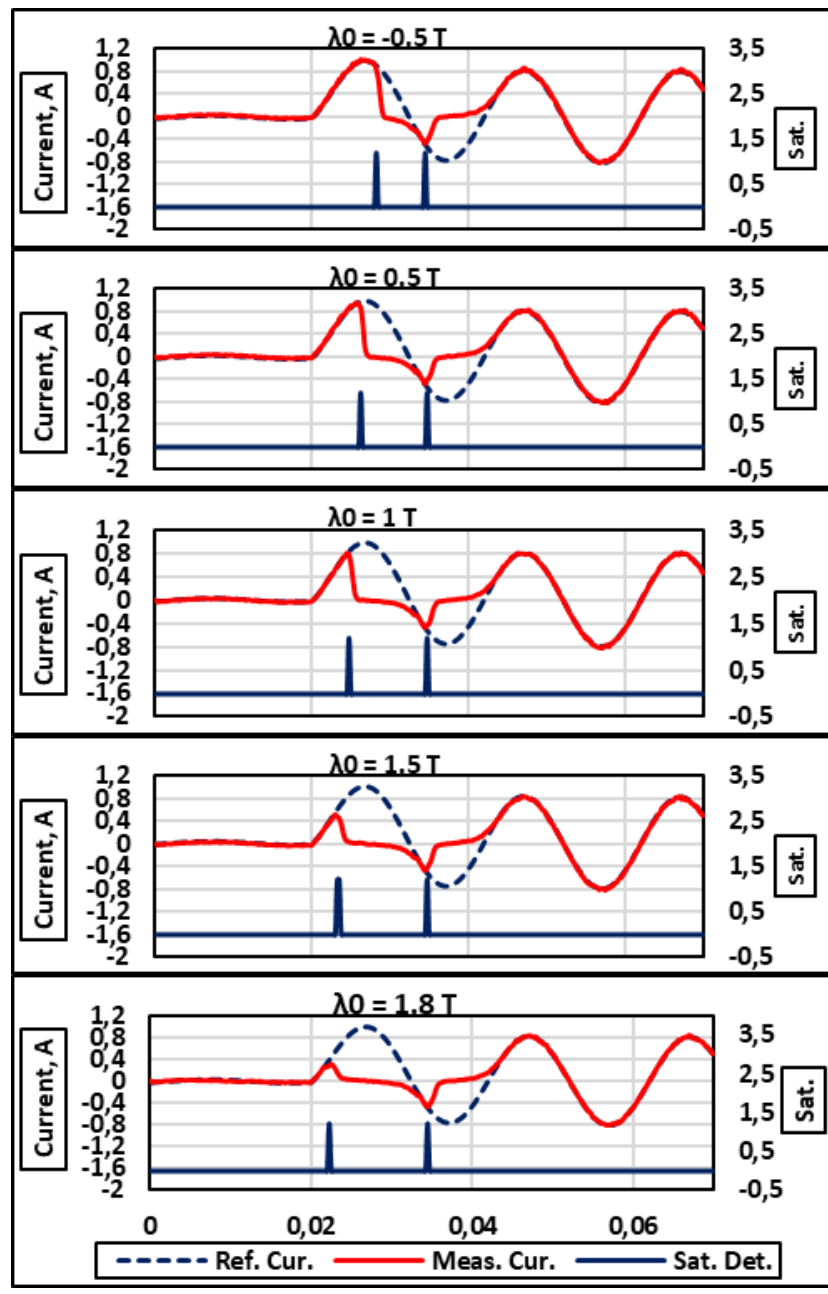

Fig. 7. CT currents and its saturation detection with variation of $\lambda_{0}$

The experimental results presented on the Fig. 7 show that the MFD method remains ability to detect the start of the CT core saturation with initial flux density and even during short US section (3-4 samples) the method still stays able to perform accurate detection of the start saturation.

\section{Conclusions}

Within the paper a new CT saturation detection method has been proposed. For correct operation of the method, a threshold calculation and the method setting are given. The method sensitivity was checked with respect to a noise and a level of the initial flux density. Difference function-based methods are analyzed. The advantages and disadvantages of the existing saturation detection methods are described.

To avoid maloperation of the method in normal and peak conditions of a grid a new amplitude prediction method has been proposed which was implemented on the basis of numerical differentiation. It is well known that the numerical differentiation has high sensitivity to noise as a result their accuracy can be significantly decreased. To reduce this effect, a median filtering of the measured current was used. Afterwards, based on 3 or 4 samples of 
the measured current related to unsaturated section the average predicted amplitude was obtained.

Experiments results of the paper show that proposed saturation detection method has a high reliability with respect to initial flux density and noise. It is clearly seen from these experiments that during severe saturation, when unsaturated section contains only 4 samples of the measured current at an 80 samples/cycle, the proposed method remains capable to detect the start of saturation.

\section{Acknowledgement}

Proposed results are the outcome of work on the topic "Development and testing algorithms and technical solutions for digital control of power facilities" within integrated project "Improvement of approaches to digital management of power facilities".

\section{References}

[1] R. Hunt, "Impact of CT errors on protective relays - Case studies and analyses," IEEE Trans. Ind. Appl., vol. 48, no. 1, pp. 52-61, 2012, doi: 10.1109/TIA.2011.2175879.

[2] F. das Guerra Fernandes Guerra and W. Santos Mota, "Current transformer model," IEEE Trans. Power Deliv., vol. 22, no. 1, pp. 187-194, 2007, doi: 10.1109/TPWRD.2006.887092.

[3] H. Dashti, M. Sanaye-Pasand, and M. Davarpanah, "Current transformer saturation detectors for busbar differential protection," Proc. Univ. Power Eng. Conf., no. 1, pp. 338-343, 2007, doi: 10.1109/UPEC.2007.4468971.

[4] M. Hossain, I. Leevongwat, and P. Rastgoufard, "A Current Transformer (CT) Saturation Detection Method for Bus Differential Protection," Clemson Univ. Power Syst. Conf. PSC 2018, no. M, pp. 1-5, 2019, doi: 10.1109/PSC.2018.8664064.

[5] R. Abd Allah, S. Moussa, E. H. Shehab-Eldin, and M. N. G. Hamed, "Advanced detection and compensation scheme for current transformers saturation," Proc. 11th Int. Middle East Power Syst. Conf. MEPCON'2006, vol. 2, pp. 481486, 2006.

[6] S. Biswal and M. Biswal, "Detection of current transformer saturation phenomenon for secured operation of smart power network," Electr. Power Syst. Res., vol. 175, no. May, p. 105926, 2019, doi: 10.1016/j.epsr.2019.105926.

[7] G. L. Macieira and A. L. M. Coelho, "Evaluation of numerical time overcurrent relay performance for current transformer saturation compensation methods," Electr. Power Syst. Res., vol. 149, pp. 55-64, 2017, doi: 10.1016/j.epsr.2017.04.005.

[8] L. Yang, J. Zhao, P. A. Crossley, and K. Li, "A current transformer saturation detection algorithm for use in current differential protection," Proc. - Int. Conf. Electr. Control Eng. ICECE 2010, vol. 3, no. 3, pp. 3142-3146, 2010, doi: 10.1109/iCECE.2010.767.

[9] G. Lin, Q. Song, D. Zhang, F. Pan, and L. Wang, "A hybrid method for current transformer saturation detection and compensation in smart grid," in The 2017 4th International Conference on Systems and Informatics (ICSAI 2017), 2017, pp. 369-374.

[10] B. M. Schettino, C. A. Duque, and P. M. Silveira, "Current-Transformer Saturation Detection Using Savitzky-Golay Filter,” IEEE Trans. Power Deliv., vol. 31, no. 3, pp. 1400-1401, 2016, doi: 10.1109/TPWRD.2016.2521327.
[11] J. Herlender, J. Iżykowski, and K. Solak, "Compensation of the current transformer saturation effects for transmission line fault location with impedance-differential relay,” Electr. Power Syst. Res., vol. 182, no. May 2019, 2020, doi: 10.1016/j.epsr.2020.106223.

[12] S. Bahari, T. Hasani, and H. Sevedi, "A New Stabilizing Method of Differential Protection Against Current Transformer Saturation Using Current Derivatives," 2020 14th Int. Conf. Prot. Autom. Power Syst. IPAPS 2020, pp. 33-38, 2019, doi: 10.1109/IPAPS49326.2019.9069379.

[13] D. Behi, M. Allahbakhshi, A. Bagheri, and M. Tajdinian, "A new statistical-based algorithm for CT saturation detection utilizing residual-based similarity index," 2017 25th Iran. Conf. Electr. Eng. ICEE 2017, pp. 1072-1077, 2017, doi: 10.1109/IranianCEE.2017.7985200.

[14] A. A. A. Etumi and F. J. Anayi, "The application of correlation technique in detecting internal and external faults in three-phase transformer and saturation of current transformer," IEEE Trans. Power Deliv., vol. 31, no. 5, pp. 2131-2139, 2016, doi: 10.1109/TPWRD.2016.2572608.

[15] T. Zheng, T. Huang, Y. Ma, Z. Zhang, and L. Liu, "Histogram-Based Method to Avoid Maloperation of Transformer Differential Protection Due to CurrentTransformer Saturation under External Faults," IEEE Trans. Power Deliv., vol. 33, no. 2, pp. 610-619, 2018 , doi: 10.1109/TPWRD.2017.2712806.

[16] C. Hong, L. Haifeng, J. Hui, P. Jianchun, and H. Chun, “A scheme for detection and assessment of current transformer saturation," Proc. - 9th Int. Conf. Meas. Technol. Mechatronics Autom. ICMTMA 2017, pp. 9093, 2017, doi: 10.1109/ICMTMA.2017.0029.

[17] J. Pan, K. Vu, and Y. Hu, "An efficient compensation algorithm for current transformer saturation effects," IEEE Trans. Power Deliv., vol. 19, no. 4, pp. 1623-1628, 2004, doi: 10.1109/TPWRD.2004.835273.

[18] Y. C. Kang, U. J. Lim, S. H. Kang, and P. A. Crossley, "Compensation of the distortion in the secondary current caused by saturation and remanence in a CT," IEEE Trans. Power Deliv., vol. 19, no. 4, pp. 1642-1649, 2004, doi: 10.1109/TPWRD.2004.835266.

[19] E. Hajipour, M. Vakilian, and M. Sanaye-Pasand, "Current-Transformer Saturation Compensation for Transformer Differential Relays," IEEE Trans. Power Deliv., vol. 30, no. 5, pp. 2293-2302, 2015, doi: 10.1109/TPWRD.2015.2411736.

[20] G. Baoming, A. T. de Almeida, and T. E. Ferreira Fernando J., "Estimation of primary current in saturated current transformer using flexible neural network," Trans. Inst. Meas. Control, vol. 28, no. 1, pp. 81-91, 2006, doi: 10.1191/0142331206tm164oa.

[21] R. W. Schafer, "What Is a Savitzky-Golay Filter? [Lecture Notes]," no. July, pp. 111-117, 2011.

[22] A. Wiszniewski, W. Rebizant, and L. Schiel, "Correction of current transformer transient performance," IEEE Trans. Power Deliv., vol. 23, no. 2, pp. 624-632, 2008, doi: 10.1109/TPWRD.2008.915832.

[23] Y. C. Kang, J. K. Park, S. H. Rang, A. T. Johns, and R. K. Aggarwal, "An algorithm for compensating secondary currents of current transformers," IEEE Trans. Power Deliv., vol. 12, no. 1, pp. 116-124, 1997, doi: 10.1109/mper.1997.560670.

[24] D. C. Jiles and D. L. Atherton, "Theory of ferromagnetic hysteresis (invited)," J. Magn. Magn. Mater., vol. 61, no. 6, pp. 48-60, 1986, doi: 10.1063/1.333582.

[25] U. D. Annakkage, P. G. McLaren, E. Dirks, R. P. Jayasinghe, and A. D. Parker, "A Current Transformer Model Based on the Jiles-Atherton Theory of Ferromagnetic Hysteresis,” IEEE Trans. Power Deliv., vol. 15, no. 1, pp. 57-61, 2000, doi: 10.1109/61.891531 